\title{
Persistence of Pneumococcal Serotype 3 in Adult Pneumococcal Disease in Hong Kong
}

\author{
Reema Subramanian ${ }^{1}$, Veranja Liyanapathirana ${ }^{1,2}$, Nilakshi Barua ${ }^{1}{ }^{1}$, Rui Sun ${ }^{3,4}$, Maggie Haitian Wang ${ }^{3}$, \\ Rita Ng ${ }^{1}$, Edmund A. S. Nelson ${ }^{5} \mathbb{C}$, David S. Hui ${ }^{6}$ and Margaret Ip ${ }^{1, *(1)}$
}

1 Department of Microbiology, Faculty of Medicine, The Chinese University of Hong Kong, Prince of Wales Hospital, Hong Kong, China; reema@link.cuhk.edu.hk (R.S.); veranjacl@pdn.ac.lk (V.L.); nilakshibarua@cuhk.edu.hk (N.B.); ritang@cuhk.edu.hk (R.N.)

2 Department of Microbiology, Faculty of Medicine, University of Peradeniya, Peradeniya 20400, Sri Lanka

3 Centre for Clinical Research and Biostatistics, Jockey Club School of Public Health and Primary Care, The Chinese University of Hong Kong, Hong Kong, China; rsun@gmail.com (R.S.); maggiew@cuhk.edu.hk (M.H.W.)

4 The Seventh Affiliated Hospital of Sun Yat-sen University, Shenzhen 518107, China

5 Department of Paediatrics, Faculty of Medicine, The Chinese University of Hong Kong, Hong Kong, China; tony-nelson@cuhk.edu.hk

6 Department of Medicine \& Therapeutics, Faculty of Medicine, The Chinese University of Hong Kong, Hong Kong, China; dschui@cuhk.edu.hk

* Correspondence: margaretip@cuhk.edu.hk; Tel.: +852-3505-1265; Fax: +852-2647-3227

Citation: Subramanian, $\mathrm{R}$

Liyanapathirana, V.; Barua, N.; Sun,

R.; Wang, M.H.; Ng, R.; Nelson,

E.A.S.; Hui, D.S.; Ip, M. Persistence of Pneumococcal Serotype 3 in Adult Pneumococcal Disease in Hong Kong. Vaccines 2021, 9, 756. https:// doi.org/10.3390/vaccines 9070756

Academic Editor: Giuseppe La Torre

Received: 4 June 2021

Accepted: 4 July 2021

Published: 7 July 2021

Publisher's Note: MDPI stays neutral with regard to jurisdictional claims in published maps and institutional affiliations.

Copyright: (c) 2021 by the authors. Licensee MDPI, Basel, Switzerland. This article is an open access article distributed under the terms and conditions of the Creative Commons Attribution (CC BY) license (https:// creativecommons.org/licenses/by/ $4.0 /)$.
Abstract: The epidemiology of hospitalised pneumococcal disease in adults following the introduction of universal childhood pneumococcal immunisation in 2009 was assessed. Culture-confirmed Streptococcus pneumoniae (SP) from adults hospitalised between 2009 to 2017 were examined. The cases were categorised into invasive pneumococcal disease (IPD) and pneumonia (bacteraemic, non-bacteraemic, and that associated with other lung conditions). The isolates were serotyped and antimicrobial susceptibilities were determined by microbroth dilution. Patient characteristics, comorbidities, and outcomes were analysed. Seven hundred and seventy-four patients (mean age, 67.7 years, $S D \pm 15.6)$ were identified, and IPD was diagnosed in 110 (14.2\%). The most prevalent serotype, 19F, was replaced by serotype 3 over time. Penicillin and cefotaxime non-susceptibilities were high at $54.1 \%$ and $39.5 \%$ (meningitis breakpoints), $19.9 \%$ and $25.5 \%$ (non-meningitis breakpoints), respectively. The overall 30-day mortality rate was $7.8 \%$ and $20.4 \%$ for IPD. Age $\geq 75$ years (OR:4.6, CI:1.3-17.0, $p<0.02$ ), presence of any complications (OR:4.1, CI:1.02-16.3, $p<0.05$ ), pleural effusion (OR:6.7, CI:1.2-39.4, $p<0.03$ ) and intensive care unit (ICU) admission (OR:9.0, CI:1.3-63.4, $p<0.03$ ) were independent predictors of 30-day mortality. Pneumococcal disease by PCV 13 covered serotypes; in particular, $19 \mathrm{~F}$ and 3 are still prominent in adults. Strengthening targeted adult vaccination may be necessary in order to reduce disease burden.

Keywords: pneumococcal disease; pneumonia; adults; risk factors; serotype 3

\section{Introduction}

Pneumonia accounted for $18.9 \%$ of all deaths in Hong Kong in 2019 and has remained the second most common cause of death after cancers since 2012 [1]. Despite the wide availability of pneumococcal vaccines, Streptococcus pneumoniae (SP) remains the commonest bacterial pathogen associated with pneumonia and represents $12 \%$ of all adult community-acquired pneumonia (CAP) with confirmed aetiologies [2].

The pneumococcal conjugate vaccine, PCV7, was included in Hong Kong's universal childhood immunisation in 2009 and switched to PCV13 in December 2011. Prior to this time, the vaccine had been available in the private sector. Invasive pneumococcal disease (IPD) was listed as a notifiable infectious disease in 2015 to enhance surveillance. The incidence of IPD has ranged from 1.7-2.9/100,000 population during this postintroduction 
period [3]. While the incidence among infants has decreased, serotype replacement has been noted, and serotype 3 disease still occurs despite this serotype being included in PCV13.

Since 2014, a single dose of PCV13 followed by a dose of pneumococcal polysaccharide vaccine (PPSV23) one year after PCV13 vaccination has been recommended for highrisk adults and elderly people $\geq 65$ years of age by the Scientific Committee on Vaccine Preventable Diseases under the Centre for Health Protection of Hong Kong. A total of $33.9 \%$ and $34.1 \%$ of the accumulative percentage of elderly people $\geq 65$ years received at least a single dose of 23-valent pneumococcal polysaccharide vaccine (23vPPV) or PCV13 in 2015-2016 and 2016-2017, respectively. In 2015-2016, elderly people $\geq 65$ years received a single dose of 23vPPV. From October 2017, an additional dose PCV13 has been offered to elderly people $\geq 65$ years who have high-risk conditions [4].

Studies have revealed that childhood immunisation has led to reduced adult pneumococcal disease through herd effects [5]. However, this is less marked in populations with risk factors [6], and disease burden based on IPD surveillance is often underestimated. The efficacy of the adult vaccine for SP is limited in preventing CAP [7]. A recent trial using PCV13 as a vaccine in adults for preventing CAP demonstrated substantial real-world protection of PCV13 against vaccine-type CAP in adults ( $\geq 65$ years) [8]. Out of 2034 participants, 3.3\% were identified as having PCV13 serotypes. However, these participants were less likely to be immunocompromised and overweight or obese than controls but were otherwise similar. The cases were less likely to have received PCV13 than controls.

Patient comorbidities have been independently linked to an increased likelihood of adverse outcomes (e.g., 30-day mortality) [9]. We thus sought to analyse the epidemiology of all culture-confirmed hospitalised pneumococcal disease in adults over a nine-year period and included all disease categories including non-bacteraemic pneumonia or those associated with other lung conditions to study the prevalent serotypes, antimicrobial susceptibilities, and characteristics of the patient groups, associated comorbidities, and outcome.

\section{Materials and Methods}

\subsection{Patients and Isolates}

Adult patients in the age group 16 years to 99 years were reviewed from cases in which SP was isolated during admission to the Prince of Wales Hospital, Hong Kong $(\mathrm{PWH})$ and fulfilled the definition for pneumococcal infection based on the diagnoses from the electronic medical records from January 2009-December 2017. PWH is a 1350-bed teaching hospital and is the major acute hospital for the New Territories East Cluster of the seven public hospital clusters served by the Hospital Authority of Hong Kong. Cases were included based on the confirmed culture of SP and availability of the isolate for serotyping and susceptibility testing. The SP were isolated from sputum $(n=670)$, tracheal aspirate $(n=12)$, blood $(n=84)$, bronchoalveolar lavage $(n=5)$ and others $(n=3)$.

Only the first episode of SP isolation from a patient each year was included. Nonduplicate pneumococcal isolates were serotyped using multiplex polymerase chain reaction (PCR) assays as recommended by the Centre for Disease Control (USA) [10,11]. Microbroth dilution based minimum inhibitory concentration (MIC) determination and interpretation was performed and interpreted according to Clinical and Laboratory Standard Institute (CLSI) guidelines [12]. Intermediate sensitive or resistant isolates were grouped as nonsusceptible. Interpretations were also made using European Committee on Antimicrobial Susceptibility Testing (EUCAST) [13] and Pharmacokinetic/pharmacodynamic (PK/PD) guidelines for comparison [14,15].

Clinical data were extracted from medical records, including the electronic patient records (EPR), after discharge. Demographic data including age, sex; administrative data including unit of admission, date of admission, date of discharge; comorbid conditions and disease outcome was collected. Community-acquired infections were defined as developing the symptoms and signs in the community or within $48 \mathrm{~h}$ after admission to PWH. Each comorbid condition and its severity were defined based on ICD 9 categories, and the Charlson comorbidity index was calculated [16]. An estimation of the burden 
of hospitalisation due to infective pneumonia between the period of 2009-2017 at PWH was also retrieved (ICD 9 diagnosis code between 481 to 485). The monthly rates of pneumococcal pneumonia were calculated by dividing against total admission due to infective pneumonia. Pneumococcal disease was broadly defined into six groups (Table 1) to illustrate the clinical characteristics (namely meningitis, bacteraemia, bacteraemic/nonbacteraemic pneumonia, pneumonia with concomitant lung conditions, and exacerbation of lung conditions). The last category was culture positive requiring treatment and with other concomitant lung condition(s), but did not indicate pneumonia in the ICD coding. This group include patients with COPD, emphysema, cancer, etc.

Table 1. Age stratified patient and disease characteristics ( $p$-values attained by Fisher's exact test or Chi-square for categorical variables and on-way ANOVA for continuous variables).

\begin{tabular}{|c|c|c|c|c|c|c|}
\hline & No. of Patients ( $N=774)$ & 16-49 Years ( $N=94)$ & 50-64 Years $(\mathrm{N}=180)$ & 65-74 Years $(\mathrm{N}=217)$ & $\geq 75$ Years $(\mathrm{N}=283)$ & $p$ Value \\
\hline \multicolumn{7}{|c|}{$n(\%)$} \\
\hline \multicolumn{7}{|c|}{ Patient Characteristics } \\
\hline & & & Sex & & & \\
\hline Males & $587(75.8)$ & $53(56.4)$ & $119(66.1)$ & $186(85.7)$ & $229(80.9)$ & $<0.001$ \\
\hline Females & $187(24.2)$ & $41(43.6)$ & $61(33.9)$ & $31(14.3)$ & $54(19.1)$ & $<0.001$ \\
\hline \multicolumn{7}{|c|}{ Comorbid Conditions } \\
\hline Chronic obstructive pulmonary disease & $198(26.2)$ & $5(5.32)$ & $14(8.00)(\mathrm{N}=176)$ & $69(33.2)(\mathrm{N}=208)$ & $110(39.7)(\mathrm{N}=277)$ & 0.014 \\
\hline $\begin{array}{c}(\mathrm{COPD})(\mathrm{N}=755) \\
\text { Asthma }\end{array}$ & $53(6.85)$ & $13(13.8)$ & $12(6.67)$ & $15(6.91)$ & $13(4.59)$ & $<0.001$ \\
\hline History of tuberculosis $(\mathrm{N}=769)$ & $87(11.3)$ & $9(9.60)$ & $9(5.05)(\mathrm{N}=178)$ & $25(11.6)(\mathrm{N}=215)$ & $44(15.6)(\mathrm{N}=282)$ & 0.006 \\
\hline Bronchiectasis & $45(5.81)$ & $1(1.06)$ & $9(5.00)$ & $15(6.91)$ & $20(7.07)$ & 0.144 \\
\hline Hypertension & $240(31.0)$ & $7(7.45)$ & $35(19.4)$ & $79(36.4)$ & $119(42.0)$ & $<0.001$ \\
\hline Ischaemic heart disease & $68(8.79)$ & $2(2.13)$ & $9(5.00)$ & $17(7.83)$ & $40(14.1)$ & $<0.001$ \\
\hline Congestive cardiac failure & $31(4.01)$ & $2(2.13)$ & $4(2.22)$ & $6(2.76)$ & $19(6.71)$ & 0.035 \\
\hline Cerebrovascular disease & $79(10.2)$ & $1(1.06)$ & $12(6.67)$ & $28(12.9)$ & $38(13.4)$ & 0.001 \\
\hline Hemiplegia or paraplegia & $20(2.58)$ & $1(1.06)$ & $3(1.67)$ & $6(2.76)$ & $10(3.53)$ & 0.474 \\
\hline Dementia & $28(3.62)$ & 0 & 0 & $3(1.38)$ & $25(8.83)$ & $<0.001$ \\
\hline Rheumatological diseases & $80(10.3)$ & $5(5.32)$ & $17(9.44)$ & $29(13.4)$ & $29(10.2)$ & 0.177 \\
\hline Presence of malignancy & $155(20.0)$ & $7(7.45)$ & $44(24.4)$ & $45(20.7)$ & $59(20.8)$ & 0.009 \\
\hline $\begin{array}{l}\text { Liver disease } \\
\text { L }\end{array}$ & $59(7.62)$ & $6(6.38)$ & $25(13.9)$ & $15(6.91)$ & $13(4.59)$ & 0.003 \\
\hline Peptic ulcer disease & $42(5.43)$ & $2(2.13)$ & $6(3.33)$ & $12(5.53)$ & $22(7.77)$ & 0.087 \\
\hline Chronic renal disease $(N=773)$ & $57(7.37)$ & $5(5.32)$ & $13(7.26)(\mathrm{N}=179)$ & $12(5.53)$ & $27(9.54)$ & 0.307 \\
\hline \multicolumn{7}{|c|}{ Charlson Comorbidity Score } \\
\hline Mean score (SD) & $2.03( \pm 2.43)$ & $0.947( \pm 1.77)$ & $2.72( \pm 2.99)$ & $2.07( \pm 2.26)$ & $2.21( \pm 2.28)$ & $<0.001$ \\
\hline No. with Score $\geq 1, n$ (\%) & $569(73.5)$ & $40(42.6)$ & $119(66.1)$ & $175(80.6)$ & $235(83.0)$ & $<0.001$ \\
\hline No. with Score $\geq 2, n(\%)$ & $329(42.5)$ & $17(18.1)$ & $73(40.6)$ & $102(47.0)$ & $137(48.4)$ & $<0.001$ \\
\hline Score $\geq 3, n(\%)$ & $200(26.2)$ & $9(9.97)$ & $48(26.8)$ & $53(24.7)$ & $90(32.6)$ & $<0.001$ \\
\hline \multicolumn{7}{|c|}{ Disease } \\
\hline \multicolumn{7}{|c|}{ Disease Characteristics } \\
\hline $\begin{array}{l}\text { Community acquired Infections (CAI) (N } \\
=771)\end{array}$ & $676(87.7)$ & $86(91.5)$ & $154(86.5)$ & $187(86.2)$ & $249(88.3)$ & 0.564 \\
\hline Invasive infections (IPD) * & $110(14.2)$ & $17(18.1)$ & $33(18.3)$ & $20(9.22)$ & $40(14.1)$ & 0.044 \\
\hline \multicolumn{7}{|c|}{ Broad disease category } \\
\hline & $14(1.81)$ & $2(2.13)$ & $5(2.78)$ & $5(2.30)$ & $2(0.71)$ & 0.356 \\
\hline Meningitis & $6(0.78)$ & $1(1.06)$ & $2(1.11)$ & $1(0.46)$ & $2(0.71)$ & 0.882 \\
\hline Pneumonia & $475(61.4)$ & $64(68.1)$ & $115(63.9)$ & $109(50.2)$ & $187(66.1)$ & 0.001 \\
\hline Bacteraemic pneumonia & $8818.52)$ & $14(21.9)$ & $25(21.7)$ & $13(11.95 .99)$ & $36(19.3)$ & 0.029 \\
\hline Non-bacteraemic pneumonia & $305(64.2)$ & $45(70.3)$ & $82(71.3)$ & $71(65.1)$ & $107(57.2)$ & 0.018 \\
\hline Pneumonia with other lung conditions & $82(17.3)$ & $5(7.8)$ & $8(7.0)$ & $25(22.9)$ & $44(23.5)$ & 0.001 \\
\hline Exacerbation of other lung conditions & $125(26.3)$ & $5(7.8)$ & $9(7.8)$ & $56(51.4)$ & $55(29.4)$ & $<0.001$ \\
\hline \multicolumn{7}{|c|}{ Disease Progression } \\
\hline Presence of any complication & $149(19.3)$ & $21(22.3)$ & $39(21.7)$ & $32(14.7)$ & $57(20.1)$ & 0.238 \\
\hline Pleural effusion & $20(2.58)$ & $5(5.32)$ & $8(4.44)$ & $3(1.38)$ & $4(1.41)$ & 0.045 \\
\hline Septic shock & $46(5.94)$ & $10(10.6)$ & $20(11.1)$ & $7(3.23)$ & $9(3.18)$ & $<0.001$ \\
\hline Respiratory failure & $96(12.4)$ & $15(16.0)$ & $19(10.6)$ & $24(11.1)$ & $38(13.4)$ & 0.514 \\
\hline Lung collapse & $9(1.16)$ & 0 & $4(2.22)$ & 0 & $5(1.77)$ & 0.097 \\
\hline Renal failure & $27(3.49)$ & $5(5.32)$ & $9(5.00)$ & $6(2.76)$ & $7(2.47)$ & 0.339 \\
\hline Lung abscess & $5(0.65)$ & $1(1.06)$ & $3(1.67)$ & $1(0.46)$ & 0 & 0.162 \\
\hline ICU admission $(\mathrm{N}=770)$ & $70(9.09)$ & $17(18.1)$ & $28(15.9)(\mathrm{N}=179)$ & $11(5.12)(\mathrm{N}=215)$ & $14(4.96)(\mathrm{N}=282)$ & $<0.001$ \\
\hline \multirow{2}{*}{\multicolumn{7}{|c|}{ Mean length of hospitalisation (days) }} \\
\hline & & & & & & \\
\hline Death at discharge & $36(4.65)$ & $2(2.13)$ & $10(5.56)$ & $8(3.69)$ & $16(5.65)$ & 0.427 \\
\hline Died within $48 \mathrm{~h}$ of admission & $10(1.29)$ & 0 & $2(1.11)$ & $2(0.92)$ & $6(2.12)$ & 0.387 \\
\hline 30-day mortality $(\mathrm{N}=765)$ & $60(7.84)$ & $1(1.08)(\mathrm{N}=93)$ & $10(5.59)(\mathrm{N}=179)$ & $15(6.98)(\mathrm{N}=215)$ & $34(12.2)(\mathrm{N}=278)$ & 0.002 \\
\hline
\end{tabular}

* 108 had invasive disease (primary bacteraemia, Meningitis, bacteraemic pneumonia) and the remaining two cases had peritonitis as per final diagnosis in the case records.

Ethical approval was obtained from the Joint Chinese University of Hong Kong-New Territories East Cluster Clinical Research Ethics Committee (CREC: 2017.448)

\subsection{Statistical Analysis}

Statistical analyses were performed using the forecast package in R [17] and Statistical Package for Social Sciences (SPSS) version 22, IBM, (Chicago, IL, USA). Univariate analysis was conducted by the Fisher's exact test or Chi-square test for categorical variables and Student's $t$-test or One-way analysis of variance (ANOVA) for continuous variables.

Multivariate analysis was performed using multiple logistic regression model. $p<0.05$ was considered statistically significant. The seasonal autoregressive integrated moving 
average (SARIMA) model was used to forecast the trend of the rate of pneumococcal pneumonia by the $\mathrm{R}$ package forecast. The model was prepared using the auto.arima function in the forecast package in $\mathrm{R}$ for fitting the best ARIMA model by searching over all possible models within the order constraints. The maximum order of the parameters used was 5, which is the default setting of the auto.arima function. Akaike's information criterion was used to select the optimal parameters for the SARIMA model. The optimised model is $\operatorname{SARIMA}(0,1,3)(1,0,0){ }_{12}$, where $(0,1,3)$ are the autoregressive order, the degree of differencing, and the moving average order, respectively, and $(1,0,0)$ are the seasonal autoregressive order, the seasonal differencing, and the seasonal moving average order, respectively and the length of the seasonality is represented in subscript (12 months in a year).

\section{Results}

\subsection{Patient Characteristics}

Clinical data of 774 adults hospitalised at PWH during the 9-year period were analysed. Five hundred eighty seven $(75.8 \%)$ of the patients were male while $24.2 \%(n=187)$ were female. The age ranged from 16 to 99 years, with a mean age of 67.7 years (SD \pm 15.6$)$. Patients were sorted into four age categories: $12.2 \%(n=94)$ between $16-49$ years; $23.3 \%$ $(n=180)$ between $50-64$ years; $28.0 \%(n=217)$ between $65-74$ years; and $36.6 \%(n=283)$ $\geq 75$ years. The demographics and the clinical characteristics are listed in Table 1 . The most common comorbidity was hypertension, which was present in $31.0 \%(n=240)$ of patients. The proportion of patients with chronic obstructive pulmonary disease (COPD), a history of tuberculosis, hypertension, ischaemic heart disease, congestive cardiac failure and dementia was higher in patients who were $\geq 75$ years. Underlying liver disease was higher in patients between 50-64 years, while the proportion of asthma was higher in patients aged 16-49 years.

\subsection{Disease and Outcomes}

Bacteraemia, meningitis, bacteraemic pneumonia and peritonitis were considered as invasive and accounted for $14.2 \%(n=110)$ of the 774 cases. Pneumonia accounted for $61.4 \%(n=475)$ of these cases, of which, non-bacteraemic pneumonia accounted for $64.2 \%(n=305)$, and pneumonia associated with other lung conditions $17.3 \%(n=82)$. The proportion of patients requiring intensive care unit (ICU) admission was $9.09 \%$ ( $n=70$ out of 770 cases) (Table 1). The proportion of patients who developed complications was 19.3\% $(n=149)$; including respiratory failure $(12.4 \%, n=96$,$) , septic shock (5.94 \%, n=46)$, renal failure $(3.49 \%, n=27)$ and pleural effusions $(2.58 \%, n=20)$. The 30 -day mortality rate was $7.84 \%$ ( $n=60$ out of 765 cases). The 30 -day mortality was $5.78 \%$ ( $n=38$ out of 657 cases) for non-invasive disease and $20.4 \%(n=22$ out of 108 cases $)$ for invasive disease $(p<0.001, \mathrm{OR}$ : $4.2,95 \%$ CI: $2.4-7.4$ ).

\subsection{Serotypes}

Out of the 772 isolates serotyped, $19 \mathrm{~F}$ was the most common type $(24.9 \%, n=192)$, followed by $3(17.5 \%, n=135)$, serogroup $6(10.1 \%, n=78),, 19 \mathrm{~A}(6.22 \%, n=48), 14(5.57 \%$, $n=43), 23 \mathrm{~F}(5.31 \%, n=41)$ and serogroup $15(4.28 \%, n=33)$ (Table 2$)$. Serotype 19F was the commonest serotype amongst the $65-74$ and $\geq 75$ age groups. Serotype 3 was the more common amongst the 16-49 and 50-64 age groups. From 2015 onwards 19F was replaced by serotype 3 as the commonest serotype. An increasing rate of serotype 3 was evident $(p<0.001)$ (Table 3). Figure 1 shows the annual changes in the distribution of the PCV serotypes of pneumococci isolated from adult disease. 
Table 2. Distribution of common serotypes/groups isolated from patients ( $p$-values by Fisher's exact test or Chi-square test).

\begin{tabular}{|c|c|c|c|c|c|c|}
\hline $\begin{array}{c}\text { Common } \\
\text { Serotype/Group }\end{array}$ & Overall N (\%) & $16-49$ Years $n(\%)$ & $50-64$ Years $n(\%)$ & 65-74 Years $n(\%)$ & $\geq 75$ Years $n(\%)$ & $p$-Value \\
\hline $19 \mathrm{~F}$ & $192(24.9)$ & $13(13.8)$ & $28(15.6)$ & $58(26.7)$ & $93(32.9)$ & $<0.001$ \\
\hline 3 & 135 (17.5) & $25(26.6)$ & $42(23.3)$ & $33(15.2)$ & $35(12.4)$ & 0.001 \\
\hline $\begin{array}{l}\text { Serogroup } 6 \\
(6 A / B / C / D)\end{array}$ & $78(10.1)$ & $9(9.58)$ & $20(11.1)$ & $24(11.1)$ & $25(8.83)$ & 0.813 \\
\hline $19 \mathrm{~A}$ & $48(6.22)$ & $9(9.58)$ & $11(6.11)$ & $16(7.37)$ & $12(4.24)$ & 0.238 \\
\hline 14 & $43(5.57)$ & $5(5.32)$ & $8(4.44)$ & $9(4.15)$ & $21(7.42)$ & 0.372 \\
\hline $23 \mathrm{~F}$ & $41(5.31)$ & $1(1.06)$ & $11(6.11)$ & $14(6.45)$ & $15(5.30)$ & 0.243 \\
\hline $\begin{array}{l}\text { Serogroup (15 } \\
\mathrm{A} / \mathrm{B} / \mathrm{C} / \mathrm{F})\end{array}$ & $33(4.28)$ & $4(4.26)$ & $10(5.56)$ & $9(4.15)$ & $10(3.53)$ & 0.774 \\
\hline Others ** & $202(26.2)$ & $28(29.8)$ & $\begin{array}{l}50(27.8) \\
\text { Vaccine related serogroups }\end{array}$ & $52(23.9)$ & $73(25.8)$ & 0.793 \\
\hline PCV 7 types & $326(42.2)$ & $25(26.6)$ & $59(32.8)$ & $96(44.2)$ & $146(51.6)$ & $<0.001$ \\
\hline PCV10 types & $330(42.8)$ & $25(26.6)$ & $59(32.8)$ & $98(45.2)$ & 148 (52.3) & $<0.001$ \\
\hline PCV13 types & $532(68.9)$ & $61(64.9)$ & $117(65.0)$ & $153(70.5)$ & $201(71.0)$ & 0.420 \\
\hline
\end{tabular}

** Others comprise of serotypes $1,4,5,7 \mathrm{~F}, 9 \mathrm{~V}$ and $18 \mathrm{C}$.

Table 3. Annual changes in the distribution of the major serotypes/groups of pneumococci isolated from adult disease ( $p$-values by Fisher's exact test or Chi-square tests).

\begin{tabular}{|c|c|c|c|c|c|c|c|c|c|c|c|}
\hline & $\begin{array}{c}\text { PCV7 } \\
\text { Serotypes } \\
(\mathrm{N}=326)\end{array}$ & $\begin{array}{c}\text { PCV10 } \\
\text { Serotypes } \\
(\mathbf{N}=330)\end{array}$ & $\begin{array}{l}\text { PCV } 13 \\
\text { Serotypes } \\
(\mathrm{N}=532)\end{array}$ & $\begin{array}{l}\text { Non PCV } \\
13 \text { Types } \\
(\mathrm{N}=242)\end{array}$ & $\begin{array}{c}19 \mathrm{~F}(\mathrm{~N}= \\
192)\end{array}$ & $\begin{array}{c}3(\mathrm{~N}= \\
135)\end{array}$ & $\begin{array}{c}19 \mathrm{~A}(\mathrm{~N}= \\
48)\end{array}$ & $\begin{array}{l}\text { Serogroup } \\
6(N=78)\end{array}$ & $\begin{array}{c}14(\mathrm{~N}= \\
43)\end{array}$ & $\begin{array}{c}23 \mathrm{~F}(\mathrm{~N}= \\
41)\end{array}$ & $\begin{array}{c}\text { Serogroup } \\
15(\mathbf{N}= \\
33)\end{array}$ \\
\hline Year $(\mathbf{N})$ & \multicolumn{11}{|c|}{ Total Isolates Per Year (\%) } \\
\hline 2009 (127) & $72(56.7)$ & $73(57.5)$ & $90(70.9)$ & $37(29.1)$ & $33(26.0)$ & $14(11.0)$ & $2(1.58)$ & $15(11.8)$ & $12(9.45)$ & $12(9.45)$ & $5(3.94)$ \\
\hline 2010 (117) & $47(40.2)$ & $48(41.0)$ & $73(62.4)$ & $44(37.6)$ & $28(23.9)$ & $13(11.1)$ & $6(5.13)$ & $18(15.4)$ & $7(5.98)$ & $5(4.27)$ & $4(3.42)$ \\
\hline $2011(121)$ & $62(51.2)$ & $62(51.2)$ & $87(71.9)$ & $34(28.1)$ & $38(31.4)$ & $16(13.2)$ & $8(6.61)$ & $9(7.44)$ & $8(6.61)$ & $7(5.79)$ & $4(3.31)$ \\
\hline $2012(93)$ & $50(53.8)$ & $51(54.8)$ & $71(76.3)$ & $22(23.7)$ & $28(30.1)$ & $19(20.4)$ & $1(1.08)$ & $12(12.9)$ & $4(4.30)$ & $9(9.68)$ & $2(2.15)$ \\
\hline $2013(81)$ & $27(33.3)$ & $27(33.3)$ & $46(56.8)$ & $35(42.3)$ & $16(19.8)$ & $9(11.1)$ & $6(7.41)$ & $9(11.1)$ & $5(6.17)$ & $3(3.70)$ & $8(9.88)$ \\
\hline $2014(69)$ & $27(39.1)$ & $27(39.1)$ & $59(85.5)$ & $10(14.5)$ & $19(27.5)$ & $18(26.1)$ & $10(14.5)$ & $6(8.70)$ & $5(7.25)$ & $3(4.35)$ & $3(4.35)$ \\
\hline 2015 (67) & $18(26.9)$ & $18(26.9)$ & $42(62.7)$ & $25(37.3)$ & $11(16.4)$ & $17(25.4)$ & $5(7.46)$ & $6(8.96)$ & $1(1.49)$ & $2(2.99)$ & $5(7.46)$ \\
\hline $2016(44)$ & $9(20.5)$ & $9(20.5)$ & $24(54.5)$ & $20(45.5)$ & $8(18.2)$ & $9(20.5)$ & $6(13.6)$ & $0(0.00)$ & $0(0.00)$ & $0(0.00)$ & $1(2.27)$ \\
\hline 2017 (55) & $14(25.5)$ & $15(27.3)$ & $40(72.7)$ & $15(27.3)$ & $11(20.0)$ & $20(36.4)$ & $4(7.27)$ & $3(5.46)$ & $1(1.82)$ & $0(0.00)$ & $1(1.82)$ \\
\hline$p$ value & $<0.001$ & $<0.001$ & 0.001 & 0.001 & 0.265 & $<0.001$ & 0.005 & 0.135 & 0.211 & 0.059 & 0.220 \\
\hline
\end{tabular}
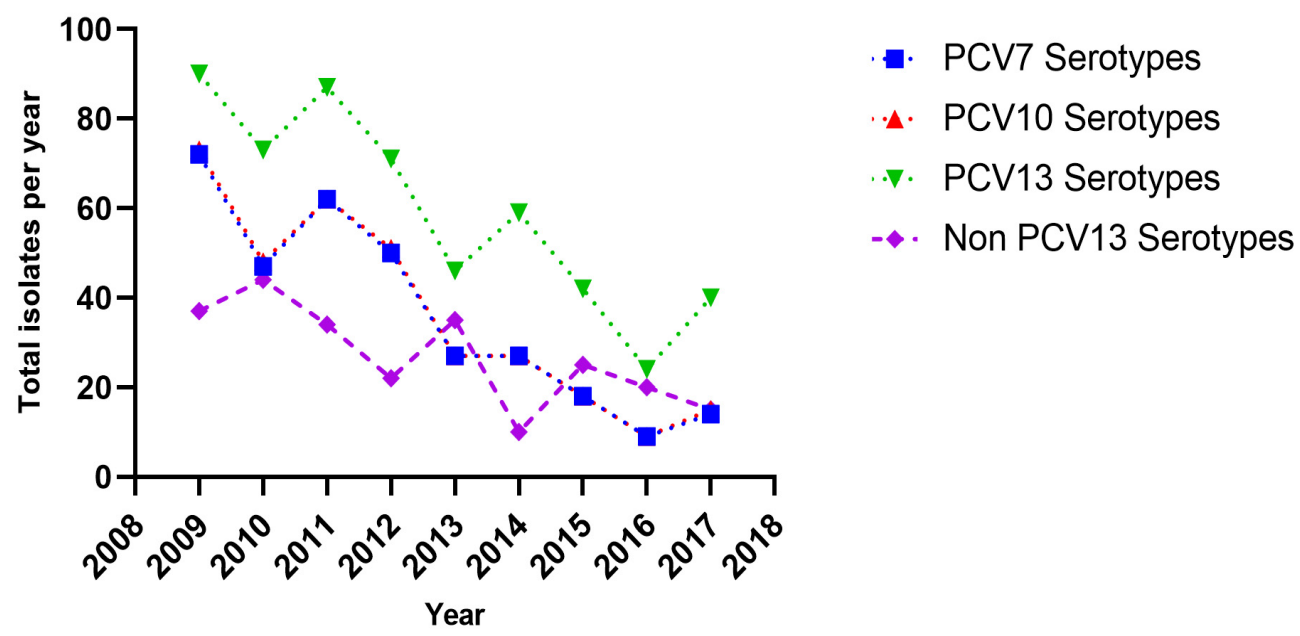

Figure 1. Annual changes in the distribution of the PCV7, PCV10, PCV13 and Non PCV13 serotypes of pneumococci isolated from adult disease.

\subsection{Antimicrobial Non-Susceptibility}

The antimicrobial susceptibilities of 764 SP isolates are listed in Table 4 . The penicillin and cefotaxime non-susceptibility rates were $19.9 \%$ and $25.5 \%$ (non-meningitis breakpoints), $54.1 \%$ and $39.5 \%$ (meningitis breakpoints), respectively. Non-susceptibilities to erythromycin, tetracycline, chloramphenicol and levofloxacin were $78.9 \%, 82.5 \%, 8.25 \%$, and $2.23 \%$, respectively. All isolates were susceptible to vancomycin and linezolid. 
Table 4. Minimum inhibitory concentrations of Streptococcus pneumoniae to 10 antibiotics $(\mathrm{N}=764)$.

\begin{tabular}{|c|c|c|c|c|c|c|c|}
\hline Antibiotic & $\begin{array}{l}\text { MIC Range } \\
(\mu \mathrm{g} / \mathrm{mL})\end{array}$ & $\mathrm{MIC}_{50}(\mu \mathrm{g} / \mathrm{mL})$ & $\mathrm{MIC}_{90}(\mu \mathrm{g} / \mathrm{mL})$ & $\begin{array}{c}\% \\
\text { Non-Susceptible } \\
(n)\end{array}$ & $\%$ Sensitive $(n)$ & $\begin{array}{c}\% \text { Intermediate } \\
(n)\end{array}$ & $\%$ Resistant $(n)$ \\
\hline Ciprofloxacin & $\leq 0.25->32$ & 1 & 2 & - & & & \\
\hline Levofloxacin & $\leq 0.25->32$ & 1 & 1 & $2.23(17)$ & $97.8(747)$ & $0.13(1)$ & $2.09(16)$ \\
\hline Lincomycin & $\leq 0.25->32$ & $>32$ & $>32$ & - & - & - & - \\
\hline Vancomycin & $\leq 0.03-1$ & 0.25 & 0.5 & 0 & $100(764)$ & - & - \\
\hline $\begin{array}{c}\text { Cefotaxime } \\
\text { (non-meningitis) }\end{array}$ & $\leq 0.015-8$ & 0.25 & 8 & 25.5 (195) & 74.5 (569) & $8.90(68)$ & $16.6(127)$ \\
\hline $\begin{array}{l}\text { Cefotaxime } \\
\text { (meningitis) }\end{array}$ & $\leq 0.015-8$ & 0.25 & 8 & $39.5(302)$ & $60.5(462)$ & $14.0(107)$ & 25.5 (195) \\
\hline $\begin{array}{c}\text { Penicillin } \\
\text { (non-meningitis) }\end{array}$ & $\leq 0.008-8$ & 0.25 & 4 & $19.9(152)$ & $80.1(612)$ & $19.5(149)$ & $0.39(3)$ \\
\hline $\begin{array}{c}\text { Penicillin } \\
\text { (meningitis) }\end{array}$ & $\leq 0.008-8$ & 0.25 & 4 & $54.1(413)$ & 45.9 (351) & & $54.1(413)$ \\
\hline Chloramphenicol & $\leq 1-32$ & 2 & 4 & $8.25(63)$ & $91.8(701)$ & - & $8.25(63)$ \\
\hline Erythromycin & $\leq 0.015->64$ & $>64$ & $>64$ & 78.9 (603) & $21.1(161)$ & $1.57(12)$ & 77.4 (591) \\
\hline Tetracycline & $0.03->32$ & 32 & $>32$ & $82.5(630)$ & 17.5 (134) & $2.49(19)$ & $80.0(611)$ \\
\hline Linezolid & $\leq 0.12-2$ & 0.5 & 1 & 0 & $100(764)$ & - & - \\
\hline
\end{tabular}

The susceptibility rates as interpreted using EUCAST breakpoints and PK/PD criteria are listed in Table 5. For both penicillin and cefotaxime, a much higher percentage of isolates will be resistant due to lower resistance breakpoint. For levofloxacin, $97.8 \%$ of the isolates were sensitive (according to PK/PD breakpoints) athough these will be non-susceptible according to EUCAST.

Table 5. Comparison of antimicrobial non-susceptibilities of Streptococcus pneumoniae using CLSI, EUCAST and PK/PD breakpoints of 764 isolates.

\begin{tabular}{|c|c|c|c|c|c|c|c|c|c|}
\hline \multirow[b]{2}{*}{ Antibiotic } & \multirow[b]{2}{*}{$\begin{array}{l}\text { MIC Range } \\
(\mu \mathrm{g} / \mathrm{mL})\end{array}$} & \multicolumn{3}{|c|}{ CLSI Breakpoints } & \multicolumn{3}{|c|}{ EUCAST Breakpoints } & \multicolumn{2}{|c|}{ PK/PD Breakpoints } \\
\hline & & $\begin{array}{c}\% \text { Sensitive } \\
(n)\end{array}$ & $\begin{array}{l}\text { \% Interme- } \\
\text { diate } \\
(n)\end{array}$ & $\begin{array}{c}\% \text { Resistant } \\
(n)\end{array}$ & $\begin{array}{c}\% \text { Sensitive } \\
(n)\end{array}$ & $\begin{array}{l}\text { \%Intermediate } \\
\text { (n) }\end{array}$ & $\begin{array}{c}\% \text { Resistant } \\
(n)\end{array}$ & $\begin{array}{c}\% \\
\text { Sensitive } \\
(n)\end{array}$ & $\begin{array}{c}\% \\
\text { Resistant } \\
(n)\end{array}$ \\
\hline Ciprofloxacin & $\leq 0.25->32$ & - & - & - & - & - & - & - & - \\
\hline Levofloxacin & $\leq 0.25->32$ & $97.8(747)$ & $0.13(1)$ & $2.09(16)$ & 0 & $97.8(747)$ & $2.23(17)$ & $97.6(746)$ & $2.36(18)$ \\
\hline Lincomycin & $\leq 0.25->32$ & - & $\begin{array}{c}0.10(1) \\
-\end{array}$ & - & - & 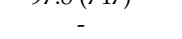 & - & 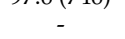 & - \\
\hline Vancomycin & $\leq 0.03-1$ & $100(764)$ & 0 & 0 & $100(764)$ & 0 & 0 & - & - \\
\hline $\begin{array}{c}\text { Cefotaxime } \\
\text { (non-meningitis) }\end{array}$ & $\leq 0.015-8$ & $74.5(569)$ & $8.90(68)$ & $16.6(127)$ & $60.5(462)$ & $22.9(175)$ & $16.6(127)$ & - & - \\
\hline $\begin{array}{l}\text { Cefotaxime } \\
\text { (meningitis) }\end{array}$ & $\leq 0.015-8$ & $60.5(462)$ & $14.0(107)$ & $25.5(195)$ & - & - & - & - & - \\
\hline $\begin{array}{c}\text { Penicillin } \\
\text { (non-meningitis) }\end{array}$ & $\leq 0.008-8$ & $80.1(612)$ & $19.5(149)$ & $0.39(3)$ & $45.9(351)$ & $34.2(261)$ & $19.9(152)$ & - & - \\
\hline $\begin{array}{c}\text { Penicillin } \\
\text { (meningitis) }\end{array}$ & $\leq 0.008-8$ & $45.9(351)$ & - & $54.1(413)$ & - & - & $54.1(413)$ & - & - \\
\hline Chloramphenicol & $\leq 1-32$ & $91.8(701)$ & - & $8.25(63)$ & $94.4(721)$ & - & $5.63(43)$ & $91.8(701)$ & $8.25(63)$ \\
\hline Erythromycin & $\leq 0.015->64$ & $21.1(161)$ & $1.57(12)$ & $77.4(591)$ & $21.1(161)$ & $1.57(12)$ & $77.4(591)$ & $21.1(161)$ & 78.9 (603) \\
\hline Tetracycline & $0.03->32$ & $17.5(134)$ & $2.49(19)$ & $80.0(611)$ & $17.5(134)$ & $2.49(19)$ & $80.0(611)$ & - & - \\
\hline Linezolid & $\leq 0.12-2$ & $100(764)$ & 0 & 0 & $100(764)$ & 0 & 0 & - & - \\
\hline
\end{tabular}

\subsection{Relationship of Serotypes with Disease/Antimicrobial Non-Susceptibilities}

Penicillin, cefotaxime, erythromycin and tetracycline non-susceptibilities had a significant association to serotypes (Table 6). Serotypes 19F and 19A had the highest rates of $\beta$-lactam non-susceptibilities of $92.7 \%$ and $87.2 \%$ to penicillin, $87.0 \%$, and $78.7 \%$ to cefotaxime (meningitis breakpoints), respectively. These serotypes were also non-susceptible to erythromycin and tetracycline. All the other serotypes were much more susceptible to $\beta$-lactams (non-meningitis breakpoints) and variable susceptibilities to erythromycin and tetracycline. On the other hand, serotype 3 isolates were susceptible to penicillin and cefotaxime at both meningitis and non-meningitis breakpoints.

The relationship between serotypes and invasive disease was statistically significant (Table 6). Invasive disease was primarily accounted for by four serotypes/groups, namely $3,15,19 \mathrm{~A}$, and 14 . The presence of complication was higher in serotypes $19 \mathrm{~A}(37.5 \%)$ and $3(28.9 \%)$ disease. Respiratory failure was higher amongst serotypes 3 and 19A disease, and pleural effusion, renal failure and septic shock were higher in serotype 3 disease. After adjusting for potential confounders, including patient age groups and patient comorbidities, 
neither of the serotypes were independently associated with the presence of complications; however, serotype 3 was independently associated with increased odds of invasive disease (OR:1.7, 95\% CI:1.0-2.7, $p=0.045$ ).

Table 6. Association between common serotypes/groups with antimicrobial non-susceptibilities and disease outcomes.

\begin{tabular}{|c|c|c|c|c|c|c|c|c|}
\hline Serotypes/Groups & $19 F(N=192)$ & $3(\mathrm{~N}=135) *$ & $6(\mathrm{~N}=78) * *$ & $14(\mathrm{~N}=43)$ & $19 \mathrm{~A} \underset{* * *}{(\mathrm{~N}}=48)$ & $23 \mathrm{~F}(\mathrm{~N}=41)$ & $15(\mathrm{~N}=33)$ & \\
\hline Outcome & & & & $n(\%)$ & & & & $p$ Value \\
\hline \multicolumn{9}{|c|}{ Antibiotic Non-Susceptibility } \\
\hline $\begin{array}{c}\text { Penicillin } \\
\text { (non-meningitis) }\end{array}$ & $118(61.5)$ & $1(0.76)$ & $1(1.32)$ & $1(2.33)$ & $26(55.3)$ & 0 & 0 & $<0.001$ \\
\hline $\begin{array}{l}\text { Penicillin } \\
\text { (meningitis) }\end{array}$ & $178(92.7)$ & $3(2.27)$ & $57(75.0)$ & $38(88.4)$ & $41(87.2)$ & $35(85.4)$ & $15(45.5)$ & $<0.001$ \\
\hline $\begin{array}{c}\text { Cefotaxime } \\
\text { (non-meningitis) }\end{array}$ & $150(78.1)$ & $1(0.76)$ & $4(5.26)$ & $3(6.98)$ & $20(42.6)$ & $9(22.0)$ & $3(9.09)$ & $<0.001$ \\
\hline $\begin{array}{l}\text { Cefotaxime } \\
\text { (meningitis) }\end{array}$ & $167(87.0)$ & $2(1.52)$ & $25(32.9)$ & $18(41.9)$ & $37(78.7)$ & $31(75.6)$ & $6(18.2)$ & $<0.001$ \\
\hline Erythromycin & $186(96.9)$ & $72(54.6)$ & $72(94.7)$ & $37(86.1)$ & $44(93.6)$ & $37(90.2)$ & $28(84.9)$ & $<0.001$ \\
\hline Tetracycline & $183(95.3)$ & $94(71.2)$ & $67(88.2)$ & $22(51.2)$ & $45(95.7)$ & $39(95.1)$ & $25(75.8)$ & $<0.001$ \\
\hline \multicolumn{9}{|c|}{ Disease outcomes } \\
\hline Invasive disease & $7(3.65)$ & $37(27.4)$ & $2(2.56)$ & $11(25.6)$ & $9(18.8)$ & $3(7.32)$ & $9(27.3)$ & $<0.001$ \\
\hline Bacteraemia & 0 & $3(2.22)$ & 0 & $2(4.65)$ & $1(2.08)$ & $1(2.44)$ & $3(9.09)$ & 0.026 \\
\hline Meningitis & $1(0.52)$ & 0 & 0 & 0 & $1(2.08)$ & 0 & 0 & 0.210 \\
\hline $\begin{array}{l}\text { Bacteraemic } \\
\text { pneumonia }\end{array}$ & $6(3.14)$ & $34(25.2)$ & $2(2.56)$ & $9(20.9)$ & $7(14.6)$ & $1(2.44)$ & $6(18.2)$ & $<0.001$ \\
\hline $\begin{array}{c}\text { Non-bacteraemic } \\
\text { pneumonia }\end{array}$ & $76(39.6)$ & $61(45.2)$ & $35(44.9)$ & 17 (39.5) & $19(39.6)$ & $18(43.9)$ & $9(27.3)$ & 0.302 \\
\hline $\begin{array}{l}\text { Pneumonia with } \\
\text { other lung conditions }\end{array}$ & $9(9.90)$ & $11(8.15)$ & $9(18.8)$ & $9(11.5)$ & $5(11.6)$ & $2(4.88)$ & $2(6.06)$ & 0.423 \\
\hline $\begin{array}{l}\text { Exacerbation of other } \\
\text { lung conditions }\end{array}$ & $42(21.9)$ & $4(2.96)$ & $7(14.6)$ & $16(20.5)$ & $3(6.98)$ & $8(19.5)$ & $6(18.2)$ & $<0.001$ \\
\hline $\begin{array}{l}\text { Presence of } \\
\text { complications }\end{array}$ & $29(15.1)$ & $39(28.9)$ & $12(15.4)$ & $7(16.3)$ & $18(37.5)$ & $5(12.2)$ & $7(21.2)$ & 0.001 \\
\hline Pleural effusions & $3(1.56)$ & $9(6.67)$ & 0 & $2(4.65)$ & $2(4.17)$ & 0 & 0 & 0.049 \\
\hline Respiratory failure & $20(10.4)$ & $29(21.5)$ & $9(11.5)$ & $4(9.30)$ & $10(20.8)$ & $4(9.76)$ & $4(12.1)$ & 0.011 \\
\hline Renal failure & $3(1.56)$ & $13(9.63)$ & $1(1.28)$ & $1(2.33)$ & $2(4.17)$ & 0 & $1(3.03)$ & 0.006 \\
\hline Septic shock & $4(2.09)$ & $19(14.1)$ & $2(2.56)$ & $2(4.65)$ & $6(12.5)$ & $2(4.88)$ & $2(6.06)$ & $<0.001$ \\
\hline ICU admissions & $4(2.08)$ & $29(21.5)$ & $6(7.69)$ & $4(9.30)$ & $10(20.8)$ & $2(4.88)$ & $2(6.06)$ & $<0.001$ \\
\hline Died within $48 \mathrm{~h}$ & 0 & $3(2.22)$ & 0 & 0 & $2(4.17)$ & 0 & $6(6.06)$ & 0.061 \\
\hline 30 day mortality **** & $16(8.51)$ & $8(6.02)$ & $3(3.85)$ & 0 & $8(16.7)$ & $2(4.88)$ & $4(12.1)$ & 0.096 \\
\hline
\end{tabular}

* Antibiogram for 132 serotype 3 isolates available; ${ }^{* *}$ Antibiogram for 76 serogroup 6 isolates available ${ }^{* * *}$ Antibiogram for 47 serotype $19 \mathrm{~A}$ isolates available ${ }^{* * * *}$ missing data (4 serotype $19 \mathrm{~F}$ cases and 2 serotype 3 cases).

\subsection{Thirty-Day Mortality, Patient and Organism Related Factors}

The age groups, clinical parameters, comorbidities, common serotypes (Table 2), and antimicrobial non-susceptibilities (susceptible or non-susceptible) were analysed in relation to the 30-day mortality (Table 7). Multivariate analysis revealed that age $\geq 75$ years (OR: 4.6, 95\% CI:1.3-17.0, $p<0.02$ ), presence of any complication (OR:4.1, CI:1.02$16.3, p<0.05)$, pleural effusion (OR:6.7, CI:1.2-39.4, $p<0.03$ ) and ICU admission (OR:9.0, CI:1.3-6.4, $p<0.03$ ) were independent predictors of 30-day mortality. 
Table 7. Risk factors associated with 30-day mortality by univariate and multivariate analysis.

\begin{tabular}{|c|c|c|c|c|}
\hline \multirow{2}{*}{ Factor } & \multirow{2}{*}{ Odds Ratio (95\% CI) } & \multirow{2}{*}{$\begin{array}{c}\text { Univariate Analysis } \\
p \text { Value }\end{array}$} & \multirow{2}{*}{ Odds Ratio (95\% CI) } & \multirow{2}{*}{$\frac{\text { Multivariate Analysis }}{p \text { Value }}$} \\
\hline & & & & \\
\hline Age $\geq 49$ years & $8.86(1.21-6.47)$ & 0.010 & & \\
\hline Age $\geq 65$ years & $2.62(1.34-5.13)$ & 0.004 & & \\
\hline Age $\geq 75$ years & $2.47(1.45-4.21)$ & 0.001 & $4.61(1.25-17.0)$ & 0.022 \\
\hline Invasive disease & $4.17(2.35-7.38)$ & $<0.001$ & & \\
\hline Bacteraemic pneumonia & $3.64(1.97-6.73)$ & $<0.001$ & & \\
\hline Meningitis & $12.3(2.43-62.4)$ & 0.008 & & \\
\hline $\begin{array}{l}\text { Exacerbation of existing lung } \\
\text { conditions }\end{array}$ & $0.166(0.04-0.691)$ & 0.005 & & \\
\hline Presence of complications & $5.43(3.15-9.35)$ & $<0.001$ & $4.08(1.02-16.3)$ & 0.046 \\
\hline Pleural effusion & $4.18(1.47-11.9)$ & 0.016 & $6.77(1.17-39.4)$ & 0.033 \\
\hline Septic shock & $3.38(1.54-7.41)$ & 0.005 & & \\
\hline Respiratory failure & $3.18(1.73-5.84)$ & $<0.001$ & & \\
\hline Lung collapse & $6.13(1.49-25.1)$ & 0.027 & & \\
\hline Renal failure & $4.77(1.92-11.9)$ & 0.003 & & \\
\hline ICU admission & $3.20(1.63-6.27)$ & $<0.001$ & $9.01(1.28-63.4)$ & 0.027 \\
\hline $\begin{array}{c}\text { Intubation/ventilatory } \\
\text { support }\end{array}$ & $2.49(1.13-6.27)$ & 0.027 & & \\
\hline Serotype 19A & $2.56(1.14-5.75)$ & 0.045 & & \\
\hline $\begin{array}{l}\text { Charlson comorbidity score } \\
\quad \geq 2\end{array}$ & $3.22(1.83-5.66)$ & $<0.001$ & & \\
\hline $\begin{array}{c}\text { Charlson comorbidity score } \\
\geq 3\end{array}$ & $2.35(1.37-4.03)$ & 0.002 & & \\
\hline Presence of malignancy & $2.98(1.72-5.16)$ & $<0.001$ & & \\
\hline Dementia & $3.62(1.83-5.66)$ & $<0.001$ & & \\
\hline Congestive cardiac failure & $2.37(0.877-6.43)$ & 0.087 & & \\
\hline $\begin{array}{l}\text { Hospitalisation within } 3 \\
\text { months prior to admission }\end{array}$ & $2.55(1.49-4.37)$ & $<0.001$ & & \\
\hline
\end{tabular}

\subsection{Trends in Hospitalisation Rates Due to Pneumococcal Pneumonia}

The SARIMA model was prepared to forecast pneumococcal pneumonia hospitalisation, which accounted for $61.4 \%$ of the pneumococcal disease in PWH for the span of the 9 -year period. Figure 2 shows the time series of pneumococcal pneumonia hospitalisation with respect to all causes infective pneumonia at $\mathrm{PWH}$ and trends predicted using the SARIMA model. The mean rate of hospitalisation due to pneumococcal pneumonia declined from 0.209 (SD \pm 0.136 ) prior to adult PCV13 recommendation (on or before December 2014) and 0.060 (SD \pm 0.051 ) after the recommendation (after December 2014) $(p<0.001)$. From the time series data, using the SARIMA model, the predicted rate of pneumococcal pneumonia hospitalisation for the following five years (2018-2022) was also derived. The rate of pneumococcal pneumonia hospitalisation from 2016 to 2017 and the predicted monthly rate for the following five years (2018-2022) show a steady downward trend, with seasonal changes apparent. Two seasonal peaks were present, with a major peak occurring during the winter months (December-February) and a minor summer peak (July), coinciding with the local influenza peaks. 


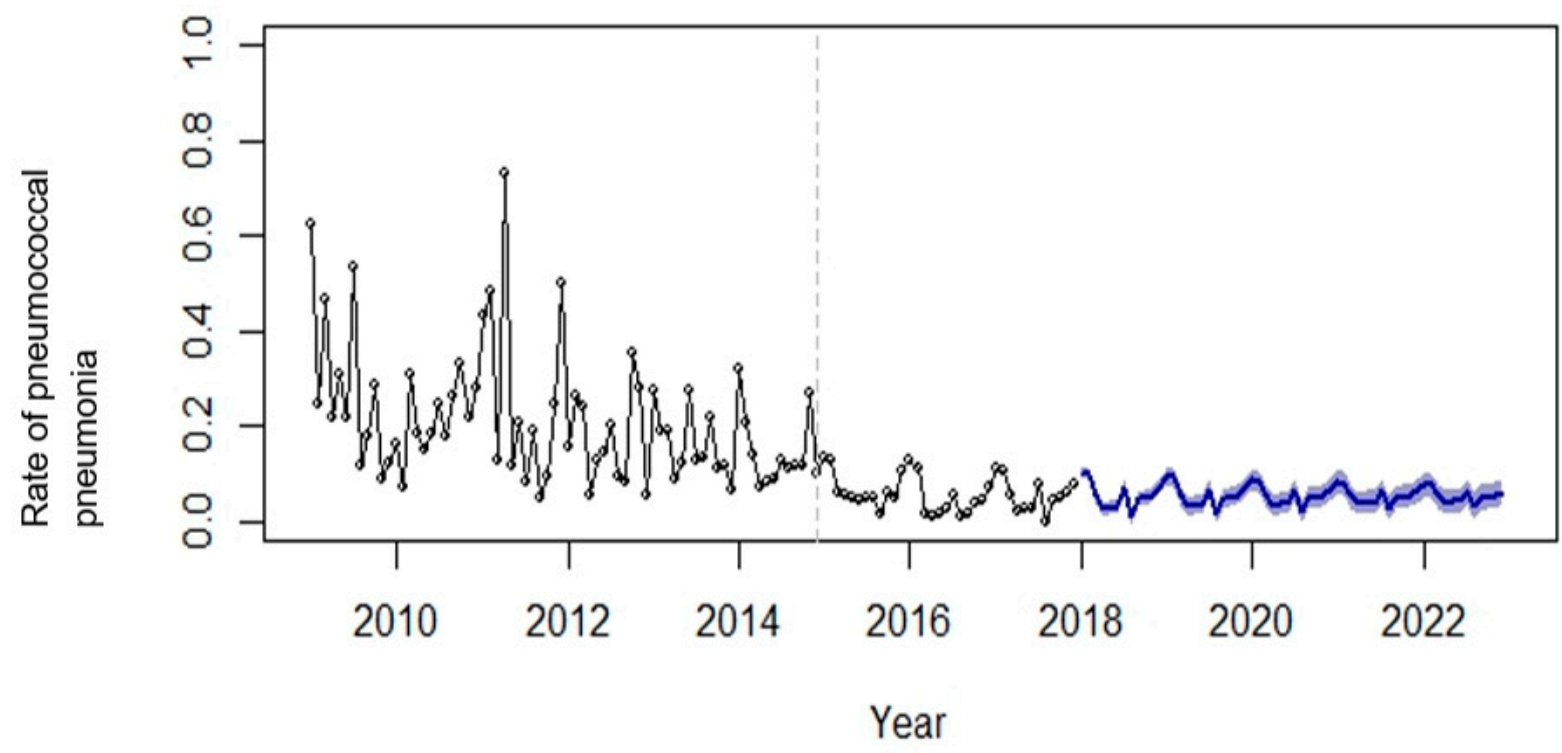

Figure 2. Monthly rate of pneumococcal pneumonia among hospitalised adults at PWH (2009-2017) and projected rates of pneumococcal pneumonia (2018-2022) using SARIMA time series model. Black points represent rates of pneumococcal pneumonia calculated from historical data. Dark blue lines represent projected rates of pneumococcal pneumonia for the following five years. The light blue region represents a 50\% confidence interval of the projected rate. The grey dashed line indicates recommendation of PCV13 for high risk adults in Hong Kong (December 2014).

\section{Discussion}

Our data revealed that six of the sixteen comorbid factors, including COPD, a history of tuberculosis, hypertension, ischemic heart disease, congestive cardiac failure, and dementia, were commonly identified in our patients with pneumococcal disease. Individuals with chronic lung and cardiovascular conditions are considered at high risk for pneumococcal disease and are recommended for vaccination. However, hypertension without complications was not considered high risk. In addition, while dementia has not been explicitly listed as a risk factor for pneumococcal disease, there is evidence that people with dementia may be at a greater risk of complications from respiratory infections, including pneumococcal disease [18].

The 30-day mortality due to IPD reported from several other countries ranges from $14 \%$ to $20 \%$ [19-21] which is comparable to that of our IPD (20.4\%). The overall 30-day mortality was $7.84 \%$. The ICU admission rate was higher among the two younger age groups. This difference may be attributed to ICU admission policies; however, more complications with septic shock and pleural effusion among the younger patients were identified, thus warranting higher ICU admissions. Differences in the proportions of serotypes in the different age groups may also contribute to the apparent higher proportion of invasive disease and ICU admission among the age groups 16-49 yrs and 50-64 yrs. Serotype 3 was highest amongst the these age groups, which on its own, was independently associated with increased odds of invasive disease.

While serotype 19F was mainly associated with patients $\geq 65$ years, from 2015 onwards, serotype 3 has replaced $19 \mathrm{~F}$ as the most common serotype overall. There have been other reports of serotype 3 remaining the leading cause of invasive disease in adults, despite childhood PCV13 immunisation [22,23]. The persistence of serotypes 19F and the rise of serotype 3 in disease indicate that the herd effect of childhood vaccination may not be as apparent, thus prompting the strengthening of targeted adult vaccination. A Canadian study reported similar findings, where vaccine-covered serotypes still posed a significant burden in adult community-acquired pneumonia as well as invasive disease despite childhood PCV13 vaccination [24]. The increasing rate of serotype 3 related disease 
locally may be due to clonal expansion, contributing to its survival and persistence despite vaccine pressure and its ability to cause more severe disease.

Furthermore, PCV13 may not be as effective in protecting against serotype 3 disease compared to other serotypes [25]. Serotype 3 may release its capsule, which is not covalently linked to peptidoglycan, thereby evading antibody-mediated protection [26]. In addition, these strains were often susceptible to $\beta$-lactam agents. This is consistent with previous findings [27]. While generally comparable, some discrepancies between CLSI and EUCAST breakpoints exist, as exemplified by susceptibilities to a penicillin (non-meningitis) and levofloxacin. Such discrepancies may be problematic, especially for the comparison of data between different regions.

The herd effect of childhood vaccination on adults may be jeopardised due to an increase in disease by non-vaccine serotypes, as exemplified in a recent study [28]. In the current cohort, serogroup 15 was common among non-PCV13 types in adult disease. With previous reports on the increase in carriage of serogroup 15 isolates in children from $[29,30]$ it is evident that serogroup 15 is circulating within the community and is likely to increase in adult disease in the future. Vaccines with expanded serotype coverage, such as the PCV20 (PCV13 types + serotypes 8, 10A, 11A, 12F, 15B, 22F, 33F) has successfully undergone phase 2 trial in adults and has exhibited promising opsonophagocytic activity (OPA) geometric mean fold rises (GMFRs) in functionality antibody from baseline to 1 month after vaccination. PCV20 has shown a GMFR of 37.1, 49.3, 11.2, 113.4, 57.1, 55.4 and 14.0 against serotype $8,10 \mathrm{~A}, 11 \mathrm{~A}, 12 \mathrm{~F}, 15 \mathrm{~B}, 22 \mathrm{~F}$ and 33F, respectively. Therefore, PCV20 may be necessary to tackle the anticipated rise in disease by non-PCV13 types [31].

The rate of pneumococcal pneumonia hospitalisation showed a downward trend from 2009-2017, possibly reflecting the combination of the herd effect of routine childhood PCV vaccination implemented in 2009 and the direct impact of adult PCV13 in the latter years.

\section{Conclusions and Implications}

In conclusion, despite routine childhood vaccination, PCV13 type pneumococcal disease, particularly serotype 3 disease, remains a burden in the adult population. Disease caused by other serotypes covered by PCV13 remains prevalent within this adult cohort. High rates of serotype $19 \mathrm{~F}$ disease remain and are mainly responsible for non-invasive disease among the older age groups. Nonetheless, the high antimicrobial non-susceptibilities within this serotype are a cause for concern as it narrows treatment options for the elderly. High percentages of $\beta$-lactam and multidrug resistance in pneumococci have similarly been reported from the Asia Pacific region recently [32]. Publicly funded pneumococcal vaccines have been made available for eligible persons and elderly people $\geq 65$ years since October 2017 to enhance vaccine uptake in Hong Kong. Targeted adult vaccination with expanded serotypes vaccines may be a promising approach in reducing the burden of adult pneumococcal disease.

Author Contributions: R.S. (Reema Subramanian) conducted the microbiological investigations, extracted patient clinical data, performed analyses of the microbiological and clinical data, drafted the initial manuscript and approved the final manuscript as submitted. V.L. and N.B. conducted the microbiological investigations, extracted patient clinical data, performed analyses of the microbiological and clinical data and approved the final manuscript as submitted. R.S. (Rui Sun) and M.H.W. constructed and interpreted the time series model, reviewed, revised and approved the final manuscript as submitted. R.N. extracted patient clinical data reviewed, revised and approved the final manuscript as submitted. E.A.S.N. and D.S.H. conceptualised the study, reviewed, revised and approved the final manuscript as submitted. M.I. conceptualised the study, supervised the microbiological investigations and clinical data collections, carried out data interpretation, drafted the initial manuscript and approved the final manuscript. All authors have read and agreed to the published version of the manuscript.

Funding: This study was supported as investigator-initiated research from Pfizer Corp (Hong Kong) Ltd. (PIM.I., Grant number TP1712042). 
Institutional Review Board Statement: The study was conducted according to the guidelines of the Declaration of Helsinki, and approved by the Joint Chinese University of Hong Kong-New Territories East Cluster Clinical Research Ethics Committee of The Chinese University of Hong Kong (CREC: 2017.448) and approved on 14 September 2017.

Informed Consent Statement: Patient consent was waived as the study was retrospective and data was retrieved from clinical records.

Data Availability Statement: The data presented in this study are available on request from the corresponding author. The data are not publicly available due to privacy and ethical restrictions.

Conflicts of Interest: All the authors have indicated they have no potential conflict of interest to disclose.

Financial Disclosure: E.A.S.N. participated in a Scientific Input Engagement meeting on the dynamic transmission modelling of rotavirus transmission and the impact of rotavirus vaccination organised by Merck Sharp \& Dohme (Asia) Ltd. in September 2019. Honorarium paid to E.A.S.N.'s Institution. M.I. currently receives funds from Pfizer for a study on adult pneumococcal diseases. The other authors have indicated they have no financial relationships relevant to this article to disclose. M.I. has received funds from Pfizer Corp Ltd., GSK and MSD in an advisory or consultancy role and/or as speaker at meetings on respiratory pathogens and infections.

\section{References}

1. Centre for Health Protection, Department of Health Hong Kong. Death Rates by Leading Causes of Death, 2001-2019. Available online: https:/ / www.chp.gov.hk/en/statistics/data/10/27/117.html (accessed on 3 June 2021).

2. Lui, G.; To, H.K.W.; Lee, N.; Chan, R.W.Y.; Li, T.; Wong, R.Y.K.; Hui, D.S.C.; Ip, M. Adherence to Treatment Guideline Improves Patient Outcomes in a Prospective Cohort of Adults Hospitalized for Community-Acquired Pneumonia. Open Forum Infect. Dis. 2020, 7, 146. [CrossRef] [PubMed]

3. Chan, D. Review of Pneumococcal Vaccination and Invasive Pneumococcal Disease in Hong Kong; Communicable Disease Watch, Centre for Health: Hong Kong, China, 2018; Volume 15, pp. 4-5. Available online: https://www.chp.gov.hk/files/pdf/cdw_v15_1.pdf (accessed on 15 May 2021).

4. Available online: Pneumococcal vaccination:People aged 65 years or above under the Government Vaccination Programme and the Vaccination Subsidy Scheme. Available online: https://www.chp.gov.hk/en/statistics/data/10/100044/6870.html (accessed on 3 June 2021).

5. Scelfo, C.; Menzella, F.; Fontana, M.; Ghidoni, G.; Galeone, C.; Facciolongo, N.C. Pneumonia and Invasive Pneumococcal Diseases: The Role of Pneumococcal Conjugate Vaccine in the Era of Multi-Drug Resistance. Vaccines 2021, 9, 420. [CrossRef] [PubMed]

6. Griffin, M.R.; Zhu, Y.; Moore, M.R.; Whitney, C.G.; Grijalva, C.G. U.S. Hospitalizations for Pneumonia after a Decade of Pneumococcal Vaccination. NEJM 2013, 369, 155-163. [CrossRef] [PubMed]

7. Moberley, S.; Holden, J.; Tatham, D.P.; Andrews, R.M. Vaccines for preventing pneumococcal infection in adults. Cochrane Database Syst. Rev. 2013, 2013, CD000422. [CrossRef] [PubMed]

8. McLaughlin, J.M.; Jiang, Q.; Isturiz, R.E.; Sings, H.L.; Swerdlow, D.L.; Gessner, B.D.; Carrico, R.M.; Peyrani, P.; Wiemken, T.L.; Mattingly, W.A.; et al. Effectiveness of 13-valent pneumococcal conjugate vaccine against hospitalization for community-acquired pneumonia in older US adults: A test-negative design. Clin. Infect. Dis. 2018, 67, 1498-1506. [CrossRef] [PubMed]

9. Said, M.A.; Johnson, H.L.; Nonyane, B.A.S.; Deloria-Knoll, M.; Brien, K.L.; Andreo, F.; Beovic, B.; Blanco, S.; Boersma, W.G.; Boulware, D.R.; et al. Estimating the Burden of Pneumococcal Pneumonia among Adults: A Systematic Review and Meta-Analysis of Diagnostic Techniques. PLoS ONE 2013, 8, e60273. [CrossRef] [PubMed]

10. Alanee, S.R.J.; McGee, L.; Jackson, D.; Chiou, C.C.; Feldman, C.; Morris, A.J.; Ortqvist, A.; Rello, J.; Luna, C.M.; Baddour, L.M.; et al. Association of Serotypes of Streptococcus pneumoniae with Disease Severity and Outcome in Adults: An International Study. Clin. Infect. Dis. 2007, 45, 46-51. [CrossRef]

11. Centers for Disease Control and Prevention. Protocol for Multiplex PCR—S. pneumoniae Serotyping-Clinical Specimens-Latin America Set. Available online: https://www.cdc.gov/streplab/downloads/pcr-Latin-Amer-clinical-specimens.pdf (accessed on 21 August 2014).

12. CLSI. Performance Standards for Antimicrobial Susceptibility Testing; 28th informational supplement; Clinical and Laboratory Standards Institute: Wayne, PA, USA, 2018.

13. EUCAST. Breakpoints for Interpretation of MICs and Zone Diameters. Version 10.0. 2020. Available online: http:/ / www.eucast org/fileadmin/src/media/PDFs /EUCAST_files/Breakpoints_tables/v_10.0_Breakpoint_Tables.pdf (accessed on 28 January 2021).

14. Sinus and Allergy Health Partnership. Antimicrobial treatment guidelines for acute bacterial rhinosinusitis. Otolaryngol. Head Neck Surg. 2004, 130, 1-45. [CrossRef] [PubMed] 
15. Jacobs, M.R.; Felmingham, D.; Appelbaum, P.C.; Grüneberg, R.N. The Alexander Project 1998-2000: Susceptibility of pathogens isolated from community-acquired respiratory tract infection to commonly used antimicrobial agents. J. Antimicrob. Chemother. 2003, 52, 229-246. [CrossRef] [PubMed]

16. Charlson, M.E.; Pompei, P.; Ales, K.L.; MacKenzie, C. A new method of classifying prognostic comorbidity in longitudinal studies: Development and validation. J. Chronic Dis. 1987, 40, 373-383. [CrossRef]

17. Hyndman, R.; Khandakar, Y. Automatic Time Series Forecasting: TheforecastPackage forR. J. Stat. Softw. 2008, 27, 1-22. [CrossRef]

18. Ridda, I.; Dastouri, F.; King, C.; Yin, J.K.; Tashani, M.; Rashid, H. Vaccination of Older Adults with Dementia Against Respiratory Infections. Infect. Disord. Drug Targets 2014, 14, 133-139. [CrossRef] [PubMed]

19. Harboe, Z.B.; Dalby, T.; Weinberger, D.; Benfield, T.; Mølbak, K.; Slotved, H.C.; Suppli, C.H.; Konradsen, H.B.; Valentiner-Branth, P. Impact of 13-Valent Pneumococcal Conjugate Vaccination in Invasive Pneumococcal Disease Incidence and Mortality. Clin. Infect. Dis. 2014, 59, 1066-1073. [CrossRef] [PubMed]

20. Marrie, T.J.; Tyrrell, G.J.; Garg, S.; Vanderkooi, O.G. Factors Predicting Mortality in Invasive Pneumococcal Disease in Adults in Alberta. Medicine 2011, 90, 171-179. Available online: https:/ /www.ncbi.nlm.nih.gov/pubmed/21512414 (accessed on 28 January 2021). [CrossRef] [PubMed]

21. Wagenvoort, G.H.; Sanders, E.A.; De Melker, H.E.; Van Der Ende, A.; Vlaminckx, B.J.; Knol, M.J. Long-term mortality after IPD and bacteremic versus non-bacteremic pneumococcal pneumonia. Vaccine 2017, 35, 1749-1757. [CrossRef]

22. Horácio, A.N.; Silva-Costa, C.; Lopes, J.P.; Ramirez, M.; Cristino, J.M.; Portuguese Group for the Study of Streptococcal Infections; Vaz, T.; Gião, M.; Ferreira, R.; Fonseca, A.B.; et al. Serotype 3 Remains the Leading Cause of Invasive Pneumococcal Disease in Adults in Portugal (2012-2014) Despite Continued Reductions in Other 13-Valent Conjugate Vaccine Serotypes. Front. Microbiol. 2016, 7, 1616. [CrossRef]

23. Slotved, H.-C.; Dalby, T.; Harboe, Z.B.; Valentiner-Branth, P.; de Casadevante, V.F.; Espenhain, L.; Fuursted, K.; Konradsen, H.B. The incidence of invasive pneumococcal serotype 3 disease in the Danish population is not reduced by PCV-13 vaccination. Heliyon 2016, 2, e00198. [CrossRef] [PubMed]

24. LeBlanc, J.J.; ElSherif, M.; Ye, L.; MacKinnon-Cameron, D.; Li, L.; Ambrose, A.; Hatchette, T.F.; Lang, A.L.; Gillis, H.; Martin, I.; et al. Burden of vaccine-preventable pneumococcal disease in hospitalized adults: A Canadian Immunization Research Network (CIRN) Serious Outcomes Surveillance (SOS) network study. Vaccine 2017, 35, 3647-3654. [CrossRef] [PubMed]

25. Andrews, N.J.; Waight, P.A.; Burbidge, P.; Pearce, E.; Roalfe, L.; Zancolli, M.; Slack, M.; Ladhani, S.N.; Miller, E.; Goldblatt, D. Serotype-specific effectiveness and correlates of protection for the 13-valent pneumococcal conjugate vaccine: A postlicensure indirect cohort study. Lancet Infect. Dis. 2014, 14, 839-846. [CrossRef]

26. Choi, E.H.; Zhang, F.; Lu, Y.-J.; Malley, R. Capsular Polysaccharide (CPS) Release by Serotype 3 Pneumococcal Strains Reduces the Protective Effect of Anti-Type 3 CPS Antibodies. Clin. Vaccine Immunol. 2016, 23, 162-167. [CrossRef] [PubMed]

27. Song, J.-H.; Dagan, R.; Klugman, K.P.; Fritzell, B. The relationship between pneumococcal serotypes and antibiotic resistance. Vaccine 2012, 30, 2728-2737. [CrossRef] [PubMed]

28. González-Díaz, A.; Càmara, J.; Ercibengoa, M.; Cercenado, E.; Larrosa, N.; Quesada, M.; Fontanals, D.; Cubero, M.; Marimón, J.; Yuste, J.; et al. Emerging non-13-valent pneumococcal conjugate vaccine (PCV13) serotypes causing adult invasive pneumococcal disease in the late-PCV13 period in Spain. Clin. Microbiol. Infect. 2020, 26, 753-759. [CrossRef] [PubMed]

29. Chan, C.C.; Subramanian, R.; Chong, P.; Nelson, E.A.S.; Lam, H.; Li, A.M.; Ip, M. Pneumococcal carriage in young children after introduction of PCV13 in Hong Kong. Vaccine 2016, 34, 3867-3874. [CrossRef] [PubMed]

30. Liyanapathirana, V.; Nelson, E.A.S.; Ang, I.; Subramanian, R.; Ma, H.; Ip, M. Emergence of serogroup 15 Streptococcus pneumoniae of diverse genetic backgrounds following the introduction of pneumococcal conjugate vaccines in Hong Kong. Diagn. Microbiol. Infect. Dis. 2015, 81, 66-70. [CrossRef]

31. Hurley, D.; Griffin, C.; Young, M.; Scott, D.A.; Pride, M.W.; Scully, I.L.; Ginis, J.; Severs, J.; Jansen, K.U.; Gruber, W.C.; et al. Safety, Tolerability, and Immunogenicity of a 20-Valent Pneumococcal Conjugate Vaccine (PCV20) in Adults 60 to 64 Years of Age. Clin. Infect. Dis. 2020. [CrossRef]

32. Torumkuney, D.; van, P.H.; Thinh, L.Q.; Koo, S.H.; Tan, S.H.; Lim, P.Q.; Sivhour, C.; Lamleav, L.; Somary, N.; Sosorphea, S.; et al. Results from the Survey of Antibiotic Resistance (SOAR) 2016-18 in Vietnam, Cambodia, Singapore and the Philippines: Data based on CLSI, EUCAST (dose-specific) and pharmacokinetic/pharmacodynamic (PK/PD) breakpoints. J. Antimicrob. Chemother. 2020, 75, i19-i42. [CrossRef] [PubMed] 